\title{
Correction to: DNA analysis of benign adult familial myoclonic epilepsy reveals associations between the pathogenic TTTCA repeat insertion in SAMD12 and the nonpathogenic TTTTA repeat expansion in TNRC6A
}

\author{
Akane Terasaki - Masayuki Nakamura $\cdot$ Yuka Urata $\cdot$ Hanae Hiwatashi • Izumi Yokoyama $\cdot$ Takeshi Yasuda \\ Teiichi Onuma - Kazumaru Wada - Sunao Kaneko - Rumiko Kan - Shin-ichi Niwa - Ohiko Hashimoto • \\ Osamu Komure · Yu-ichi Goto 1 - Yuko Yamagishi • Misa Nakano $\cdot$ Yoshihiko Furusawa - Akira Sano
}

Published online: 13 November 2020

(c) The Author(s), under exclusive licence to The Japan Society of Human Genetics 2020

Correction to: Journal of Human Genetics https://doi.org/10.1038/s10038-020-00855-0

There were errors in Fig. 1. The correct Fig. 1 is as below. In the caption of Figure 3, "moderate" should be deleted from the last line.

In "Results" section, under the subsection "Expansion of the TTTTA repeat in TNRC6A", "(II-4-III-3 and II-24-III14 in pedigree 1 , II-2-III-1 in pedigree 7, and I-8-II-9 and II-10 in Fig. 1)" should be corrected as "(II-4 with III-3 and
II-24 with III-14 in pedigree 1, II-2 with III-1 in pedigree 7, and I-1 with II-1, I-8 with II-9, and I-8 with II-11 in pedigree 10 in Fig. 1)".

In "Discussion" section, in the sixth paragraph, "(II4-III-3 and II-24-III-14 in pedigree 1, II-2-III-1 in pedigree 7, and I-8-II-9 and II-10 in Fig. 1)" should be corrected as "(II-4 with III-3 and II-24 with III-14 in pedigree 1, II-2 with III-1 in pedigree 7, and I-1 with II-1, I-8 with II-9, and I-8 with II-11 in pedigree 10 in Fig. 1)". 
Pedigree 1

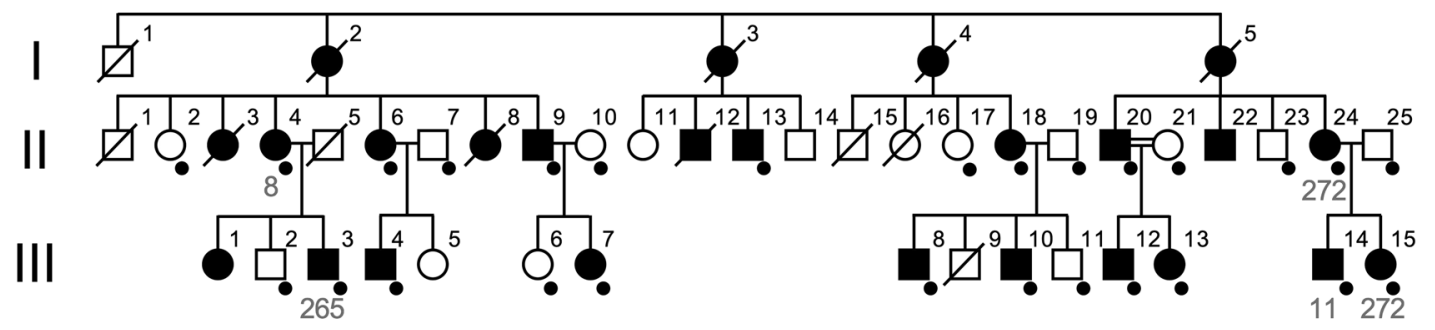

Pedigree 2

Pedigree $3 \quad$ Pedigree 4

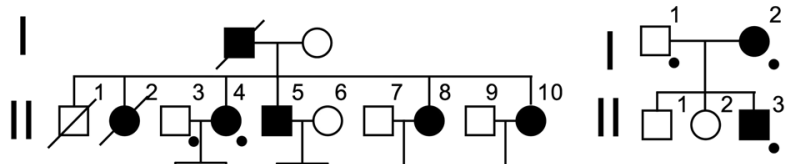

III $\square .0^{3} \square^{4} \bullet^{5} 0^{6}$

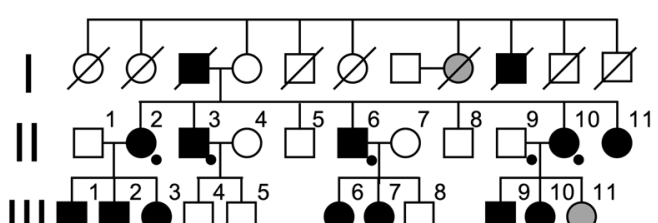

Pedigree 5

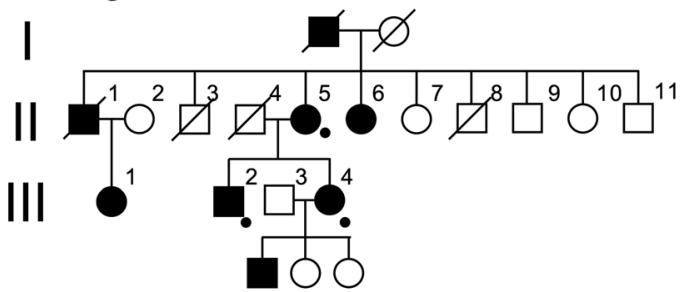

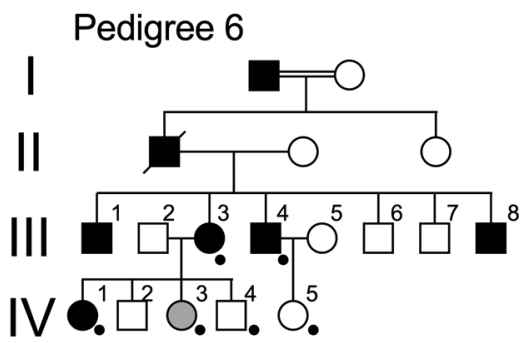

\footnotetext{
Pedigree 7

Pedigree 8

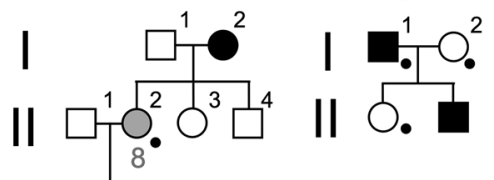

III
}

Pedigree 9

- $^{1} \mathbf{E}^{2} \mathbf{E}^{3}$

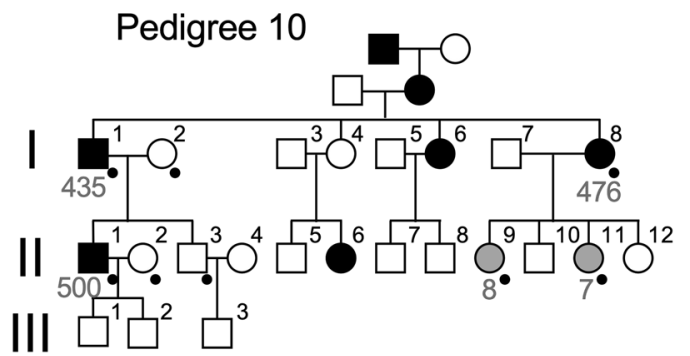

Pedigree 11

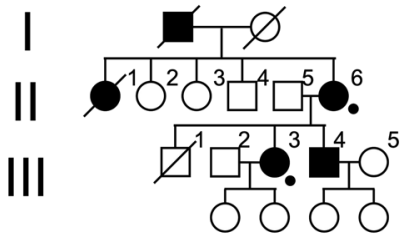

Pedigree 12

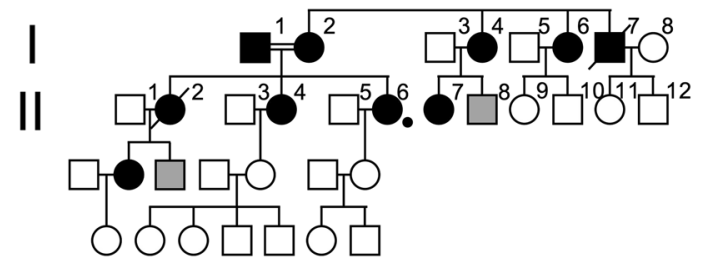

Fig. 1 